\title{
RADAR Realistic Animal Model Series for Dose Assessment
}

\author{
Mary A. Keenan ${ }^{1}$, Michael G. Stabin ${ }^{1}$, William P. Segars ${ }^{2}$, and Michael J. Fernald ${ }^{2}$
}

${ }^{1}$ Department of Radiology and Radiological Sciences, Vanderbilt University, Nashville, Tennessee; and ${ }^{2}$ Carl E. Ravin Advanced Imaging Laboratories, Duke University Medical Center, Durham, North Carolina

Rodent species are widely used in the testing and approval of new radiopharmaceuticals, necessitating murine phantom models. As more therapy applications are being tested in animal models, calculating accurate dose estimates for the animals themselves becomes important to explain and control potential radiation toxicity or treatment efficacy. Historically, stylized and mathematically based models have been used for establishing doses to small animals. Recently, a series of anatomically realistic human phantoms was developed using body models based on nonuniform rational B-spline. Realistic digital mouse wholebody (MOBY) and rat whole-body (ROBY) phantoms were developed on the basis of the same NURBS technology and were used in this study to facilitate dose calculations in various species of rodents. Methods: Voxel-based versions of scaled MOBY and ROBY models were used with the Vanderbilt multinode computing network (Advanced Computing Center for Research and Education), using geometry and tracking radiation transport codes to calculate specific absorbed fractions (SAFs) with internal photon and electron sources. Photon and electron SAFs were then calculated for relevant organs in all models. Results: The SAF results were compared with values from similar studies found in reference literature. Also, the SAFs were used with standardized decay data to develop dose factors to be used in radiation dose calculations. Representative plots were made of photon electron SAFs, evaluating the traditional assumption that all electron energy is absorbed in the source organs. Conclusion: The organ masses in the MOBY and ROBY models are in reasonable agreement with models presented by other investigators noting that considerable variation can occur between reported masses. Results consistent with those found by other investigators show that absorbed fractions for electrons for organ self-irradiation were significantly less than 1.0 at energies above $0.5 \mathrm{MeV}$, as expected for many of these small-sized organs, and measurable cross-irradiation was observed for many organ pairs for highenergy electrons (as would be emitted by nuclides such as ${ }^{32} \mathrm{P}$, ${ }^{90}$ Y, or $\left.{ }^{188} \mathrm{Re}\right)$.

Key Words: animal phantoms; internal dose assessment; nuclear medicine

J Nucl Med 2010; 51:471-476

DOI: 10.2967/jnumed.109.070532

Received Sep. 15, 2009; revision accepted Dec. 8, 2009.

For correspondence or reprints contact: Mary Ann Keenan, 1161 21st

Ave., CCC-1121 MCN, Nashville, TN 37232.

E-mail: mary.a.emmons@vanderbilt.edu

COPYRIGHT $\odot 2010$ by the Society of Nuclear Medicine, Inc. tylized and mathematically based animal models have been used in radiation dose calculations for several decades (Fig. 1). Replacement of these stylized models is now possible with realistic animal-body models based on actual CT image data. Some investigators have developed simple animal models using geometric shapes to define individual organs, as was the basis for previous human models. Hui et al. (1) developed a model based on athymic mice and calculated organ self-doses for ${ }^{90} \mathrm{Y}$. Doses from selected source or target pairs (cross-organ doses) were estimated using approximate methods based on overlapping areas of the organ pairs. Yoriyaz and Stabin (2) constructed a geometric model of the mouse and generated dose factors (DFs) for a selected number of source and target pairs for ${ }^{213} \mathrm{Bi}$ and ${ }^{90} \mathrm{Y}$. Muthuswamy et al. (3) developed a model of marrow to complement the organ model of Hui et al. (1) and provided DFs for ${ }^{131} \mathrm{I},{ }^{186} \mathrm{Re}$, and ${ }^{90} \mathrm{Y}$. Flynn et al. (4) developed a mouse model using ellipsoids to define many organs, including the kidneys, that distinguished cortex from medulla and using cylinders to define bone and marrow. Konijnenberg et al. (5) also developed a stylized representation of Wistar rats and performed Monte Carlo calculations to develop DFs for several radionuclides.

With the advent of small-animal imaging technologies, it is possible to move away from the use of stylized, equationbased body models and develop representations that more realistically define organ size, shape, and overlap. This is occurring in direct parallel with efforts to develop more realistic human models based on 3-dimensional (3D) image datasets (6). Hindorf et al. (7) developed a model of a mouse using geometric shapes to define 10 organs but treating the model in a voxel format. Figure 1 compares images of the models of Konijnenberg et al. and Hindorf et al. Kolbert et al. (8) used MR images of a female athymic mouse to develop realistic models of the kidneys, spleen, and liver and used the 3D identification code (9) to estimate self-dose and cross-dose $\mathrm{S}$ values for these organs. Stabin et al. (10) manually segmented micro-CT images of a mouse and rat and developed specific absorbed fractions (SAFs) for photon and electron sources within the animal organs and DFs for several source and target regions. 
FIGURE 1. Examples of stylized model of rodents developed by other authors: (A) Konijnenberg et al. (5) and (B) Hindorf et al. (7).

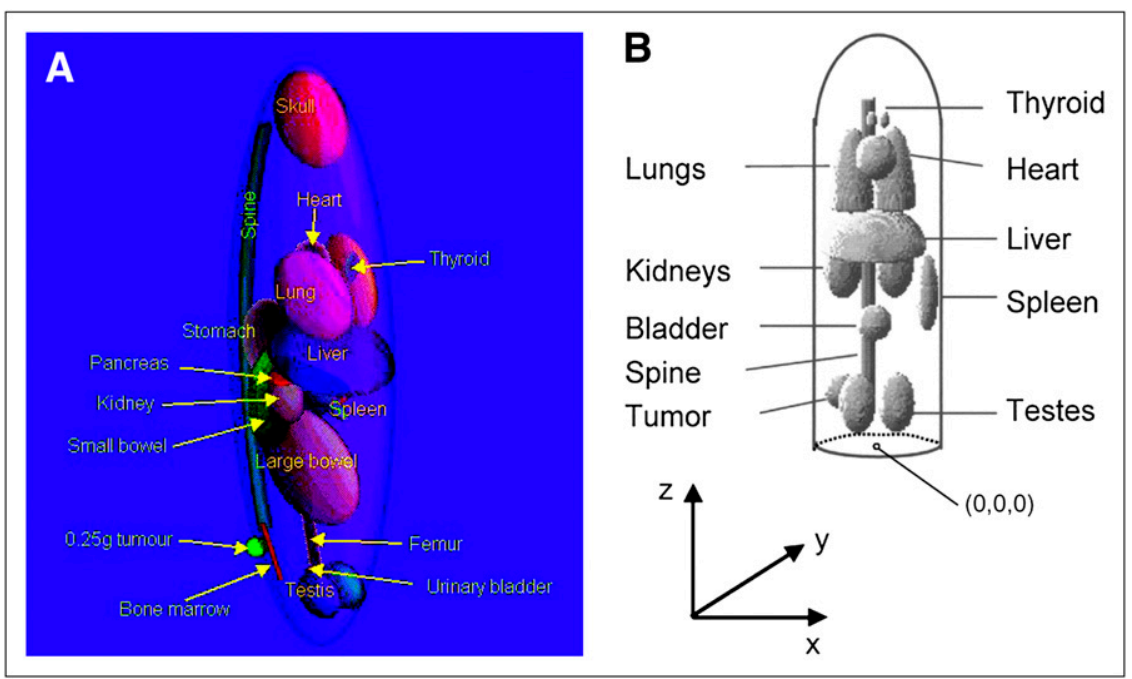

Recent work in the development of human models has focused on the use of more high-level representations (as opposed to simple geometric primitives) to define organs based on imaging data. Segars created human body models using nonuniform rational B-spline (NURBS) (11) to construct realistic and flexible organ surfaces. These models represented realistic renderings of the human body, that of both men and women, at various ages. Given the flexibility of the NURBS surfaces, computer tools developed by Segars et al. permitted rapid and easy manipulation of the organs and body (12).

Each surface can be easily altered through the set of control points that define it. Affine transformations-such as 1-dimensional, 2-dimensional, or 3D scalings-can be applied to the entire set of control points affecting the whole surface, or local deformations can be introduced by
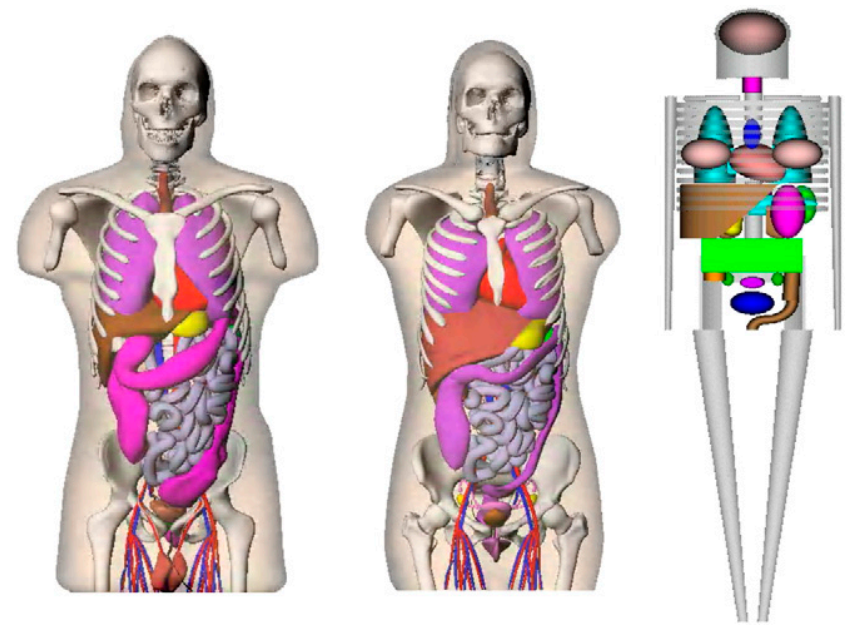

FIGURE 2. Anterior views of Segars NURBS models of adult male (A) and adult female (B) (11). (C) For comparison, stylized adult male model of Snyder et al. (24). manipulating only a handful of the control points. Figure 2 shows the adult human male and female NURBS models. The realism of these models is clearly superior to that of the stylized models of the past (Fig. 2C). Encouraged by a need for murine models to facilitate dose calculations in various species of rodents, this work was continued to create a realistic 4-dimensional digital mouse whole-body (MOBY) phantom and rat whole-body (ROBY) phantom based on the same NURBS technology (13). Figure 3 shows an image of the MOBY phantom; the realism may be compared with that of the stylized rodent models shown in Figure 1. Using mathematic, stylized models, other investigators have shown a notable effect on mouse dosimetry when geometric differences such as organ mass, organ shape, and the relative locations of organs to one

FIGURE 3. Images of unmodified ROBY and MOBY models (13), showing length of each model.

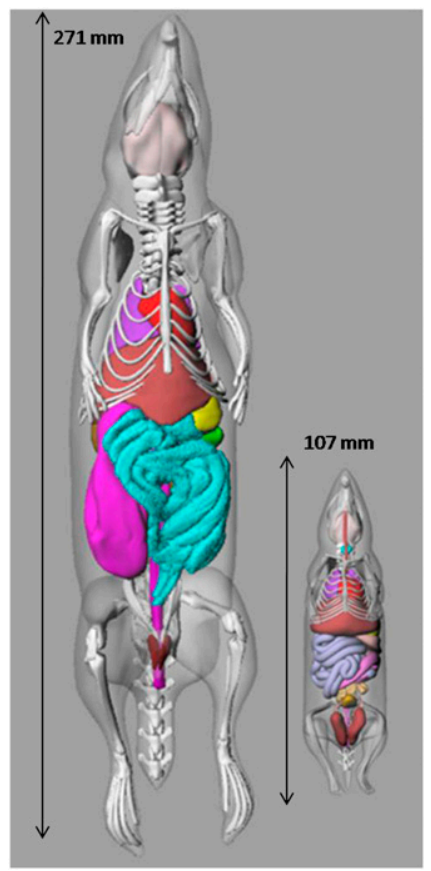


TABLE 1. Organ Masses in 3 Mouse Models

\begin{tabular}{|c|c|c|c|}
\hline \multirow[b]{2}{*}{ Organ } & \multicolumn{3}{|c|}{ Organ mass (g) } \\
\hline & 25-g mouse & 30-g mouse & 35-g mouse \\
\hline Brain & 0.466 & 0.568 & 0.666 \\
\hline Heart & 0.235 & 0.291 & 0.342 \\
\hline Stomach & 0.055 & 0.069 & 0.082 \\
\hline Small intestine & 1.74 & 2.12 & 2.49 \\
\hline Large intestine & 0.583 & 0.709 & 0.830 \\
\hline Kidneys & 0.302 & 0.374 & 0.432 \\
\hline Liver & 1.74 & 2.15 & 2.57 \\
\hline Lungs & 0.087 & 0.107 & 0.131 \\
\hline Pancreas & 0.305 & 0.378 & 0.450 \\
\hline Skeleton & 2.18 & 2.61 & 3.01 \\
\hline Spleen & 0.111 & 0.136 & 0.157 \\
\hline Testes & 0.160 & 0.197 & 0.228 \\
\hline Thyroid & 0.014 & 0.016 & 0.020 \\
\hline Bladder & 0.060 & 0.075 & 0.088 \\
\hline Body & 24.11 & 29.80 & 35.27 \\
\hline
\end{tabular}

another are considered (14). To this end, the improved realism of the MOBY and ROBY models in representing organ structure and overlap is desirable to provide DFs for use in preclinical trials in which small animals may be used and the doses to the animal organs are of interest to investigators.

\section{MATERIALS AND METHODS}

Using an interactive scaling program developed by Segars et al. (12), any NURBS model may be scaled to different sizes and shapes. One or more selected organs may be translated or rotated in any direction; scaled linearly in any direction, uniformly in 3 dimensions, or from the center by a fixed factor; or otherwise modified by the user. Instead of spending months or years creating, performing, and perfecting tedious, slice-by-slice segmentations of individual organs from diagnostic imaging data of various animals, we found this method to be much quicker, resulting in a model series that was more internally consistent. We used this program to develop a series of models representing small, medium, and large animals typically used in preclinical research in nuclear medicine-mice weighing about 25,30 , and $35 \mathrm{~g}$ and rats weighing approximately 200,300, 400, 500, and $600 \mathrm{~g}$. The resulting organ and body masses were designed to follow data found in reference literature. During radiation transport, traditionally hollow organs (e.g., stomach, intestines, heart, and bladder) were treated as a uniform organ, with mass equal to that of the wall plus contents, as defined in the NURBS models. This treatment was thought reasonable, because of uncertainties in the exact location of these small structures. The skeleton similarly

\section{TABLE 2. Organ Masses in 5 Rat Models}

\begin{tabular}{|lccccc}
\hline & \multicolumn{5}{c}{ Organ mass (g) } \\
\cline { 2 - 6 } \multicolumn{1}{c}{ Organ } & $\begin{array}{c}200-\mathrm{g} \\
\text { rat }\end{array}$ & $\begin{array}{c}300-\mathrm{g} \\
\text { rat }\end{array}$ & $\begin{array}{c}400-\mathrm{g} \\
\text { rat }\end{array}$ & $\begin{array}{c}500-\mathrm{g} \\
\text { rat }\end{array}$ & $\begin{array}{c}600-\mathrm{g} \\
\text { rat }\end{array}$ \\
\hline Brain & 1.57 & 2.32 & 3.16 & 3.93 & 4.54 \\
\hline Heart & 1.80 & 2.64 & 3.55 & 4.39 & 5.28 \\
\hline Stomach & 0.941 & 1.40 & 1.89 & 2.37 & 2.86 \\
\hline Small intestine & 10.6 & 15.5 & 20.8 & 25.6 & 30.8 \\
\hline Large intestine & 7.86 & 11.5 & 15.5 & 19.2 & 23.1 \\
\hline Kidneys & 2.06 & 3.03 & 4.09 & 5.06 & 6.09 \\
\hline Liver & 7.55 & 11.2 & 15.2 & 18.8 & 22.8 \\
\hline Lungs & 0.594 & 0.884 & 1.21 & 1.50 & 1.82 \\
\hline Pancreas & 0.368 & 0.535 & 0.732 & 0.908 & 1.10 \\
\hline Skeleton & 15.3 & 22.0 & 29.2 & 35.2 & 38.7 \\
\hline Spleen & 0.607 & 0.884 & 1.18 & 1.45 & 1.74 \\
\hline Testes & 0.174 & 0.245 & 0.321 & 0.386 & 0.460 \\
\hline Thyroid & 0.191 & 0.275 & 0.368 & 0.457 & 0.549 \\
\hline Bladder & 0.475 & 0.682 & 0.916 & 1.12 & 1.34 \\
\hline Body & 226 & 335 & 443 & 547 & 643 \\
\hline
\end{tabular}

was treated as a uniform mixture of bone, cartilage, and marrow; development of a detailed bone model with microstructure representing the individual components was beyond the scope of this project and was thought to include uncertainties similar to or greater than those for hollow organs.

Separate models were made for each size rodent. The modified models were saved, converted to a voxelized format, and used in the geometry and tracking particle transport toolkit (GEANT, version 4) (15) to perform radiation transport calculations in the voxel-based representations of the various individual models. Cubic voxels of $0.625 \mathrm{~mm}$ were used; models started at $512 \times$ $512 \times 512$ voxels but were trimmed to sizes that removed empty space around each model, to speed up the Monte Carlo simulations. For most organs, the difference between the MOBY and the ROBY reported and voxel model volumes was about $3 \%-5 \%$. For small organs, however, the difference was sometimes greater. In the absence of well-established information about these species, the tissue compositions and densities recommended for humans (16) were used for the corresponding tissues of the animals. Minor changes were suggested in the recently released revision by the International Commission on Radiological Protection (ICRP) (17). However, these changes were not deemed large enough to affect calculations from our established Monte Carlo routines, given all other uncertainties in the data and methods, which may be as much as a factor of 2 or more (18) whereas variations in tissue densities are of the order of a few percentage points. Discrete starting photon and electron energies of $0.01,0.015,0.02,0.03$, $0.05,0.1,0.2,0.5,1,1.5,2$, and $4 \mathrm{MeV}$ were simulated in available source regions. Typically 600,000 particle histories were followed in the Monte Carlo simulations, which were
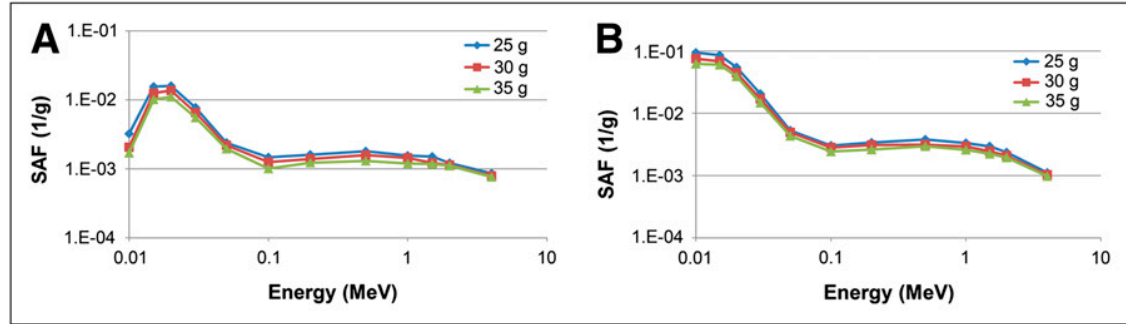

FIGURE 4. Comparison of photon SAF plots for 3 mouse models for selected organ pairs: (A) liver irradiating spleen and (B) liver irradiating lung. 
FIGURE 5. Comparison of photon SAF plots for 5 rat models for selected organ pairs: (A) liver irradiating stomach and (B) kidneys irradiating small intestine.
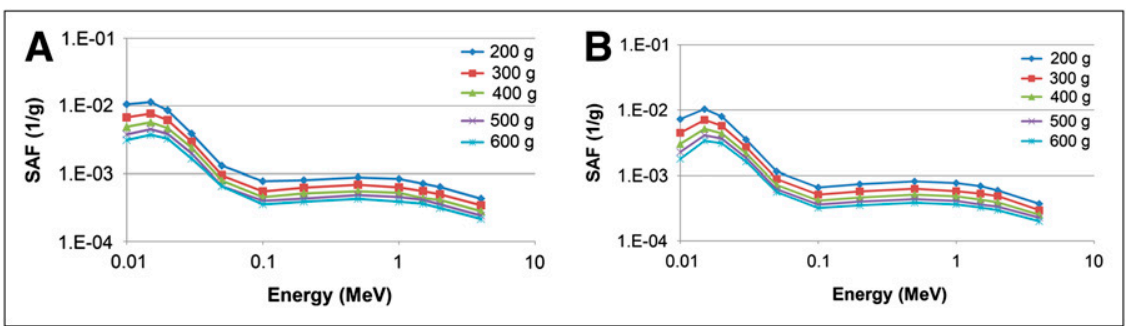

implemented on the Vanderbilt multinode computing environment (Advanced Computing Center for Research and Education). SAFs were generated for source and target regions in the models, and then organ DFs were generated, using decay data from the RAdiation Dose Assessment Resource (RADAR) (19). In most cases, uncertainties in the SAFs were under $2 \%$; in a few cases, the variability of the data was high (some small organs or organ pairs that were significantly separated), and reciprocity rules (14) and smoothing of noisy data were performed in some cases.

\section{RESULTS}

Tables 1 and 2 show final organ masses in the 3 mouse models and 5 rat models, respectively. Selected representative photon SAF plots are shown in Figures 4 and 5. Electron SAFs were also generated in this study, as the traditional assumption that all electron energy is absorbed in these small source organs is not reasonable. Electron SAFs for several organs to thyroid for the 25 -g mouse are shown in Figure 6. Table 3 shows sample DFs for ${ }^{18} \mathrm{~F}$ for the 200 -g rat model.

\section{DISCUSSION}

Most preclinical work with radiopharmaceuticals is designed to provide information about the biokinetics of new compounds for extrapolation to humans so that the radiation doses to be expected in humans can be estimated before data from clinical studies are available. Such dose estimates are only rough, as extrapolation of data from one species to another is not an exact science (20). However, in the development of some agents for therapy, radiation doses

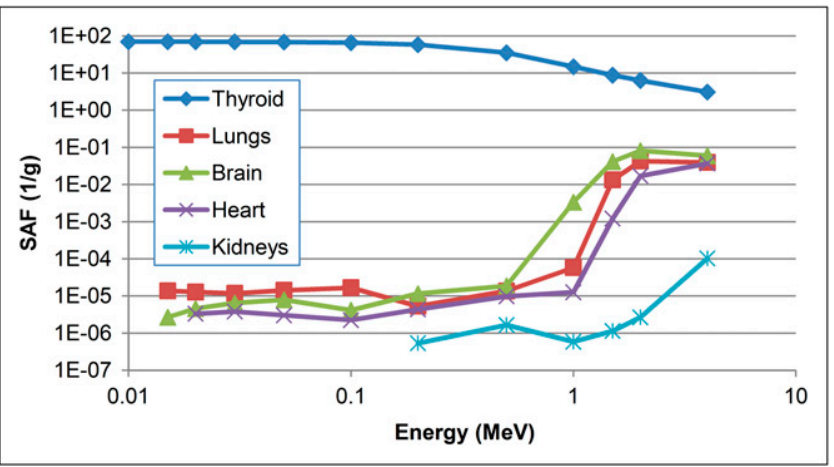

FIGURE 6. Electron SAF plots for various organs irradiating thyroid in 25-g mouse model. received by the animals are also of interest. Modification of human or animal NURBS models via spatial scaling and deformation allows for the rapid development of new models that can be adapted to specific applications and accurately tailored to meet design specifications. In this project, we did not have established reference organ masses for the different-sized rodent models that were developed, unlike the situation for reference humans, for which the ICRP has undergone extensive research and suggested many organ masses for several reference individuals (17). A wide variety of literature has reported organ masses for rodents of different sizes, and reported organ masses can vary significantly. The MOBY and ROBY models are reasonable and well-designed heterogeneous models for use in internal or external dose assessment. Radiation transport using Monte Carlo techniques is a well-established and reliable science and has been implemented in several widely used codes, including the electron gamma shower code series (21), the Monte Carlo N-particle code series (22), and GEANT (15). SAFs and DFs developed with these techniques have been widely accepted and used for standardized dosimetry in humans for many years. The extension of these techniques to dose calculations in animal models is useful in certain preclinical studies.

The organ masses in the MOBY and ROBY models are in reasonable agreement with those in models presented by other investigators (3-10), although considerable variation can occur between reported results. As found consistently by other investigators, absorbed fractions for electrons for organ self-irradiation were significantly less than 1.0 for many organs at energies above $0.5 \mathrm{MeV}$ (Fig. 6), and measurable cross-irradiation was observed for many organ pairs for high-energy electrons (as would be emitted by nuclides such as ${ }^{32} \mathrm{P},{ }^{90} \mathrm{Y}$, or ${ }^{188} \mathrm{Re}$ ).

In this model, separate bone and marrow regions were not defined. The reported DFs for the skeleton may be used as reasonable estimates to predict dose to the marrow or to dividing bone cells. No comprehensive model for the skeleton in rodent species has been defined. As in humans, this is a significant and separate modeling effort not well treated by macroscopic geometric models such as the one presented in this work. The intestines in the MOBY and ROBY models are defined more simply than in current human gastrointestinal tract models (23). No attempt was made to further segment other regions of the intestine or to differentiate wall from contents. The dose averaged over the general intestinal regions should be reasonably representative 


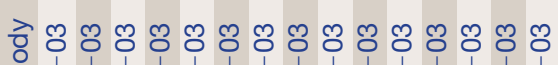
ฮ " ๘ ர

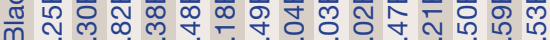
光

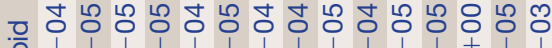
乏. ช

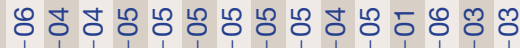
苟

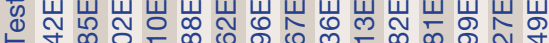

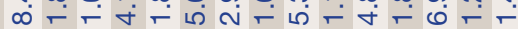

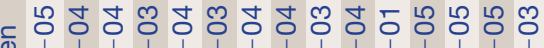
凹 山山山س山ш

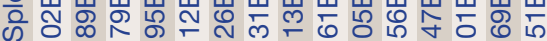
m

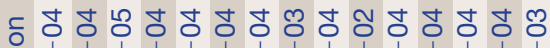

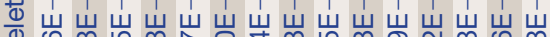

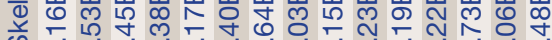
क ळ ๘

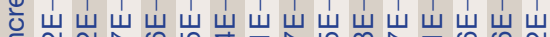
ᄃ จ 口 लं

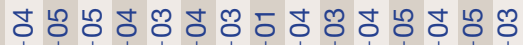
こ

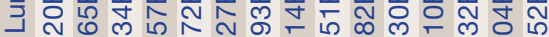

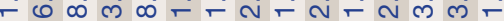

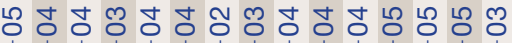

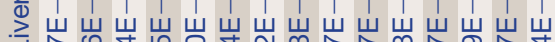

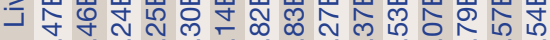
ナ

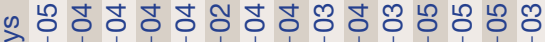
등

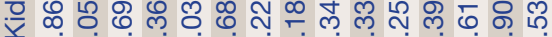
ง

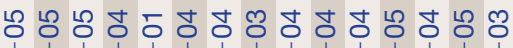
๘

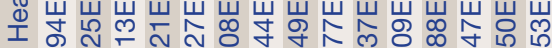
め ம்

テิ ๗ш ต స

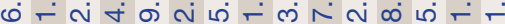

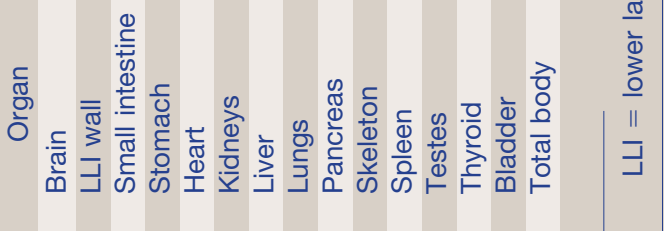

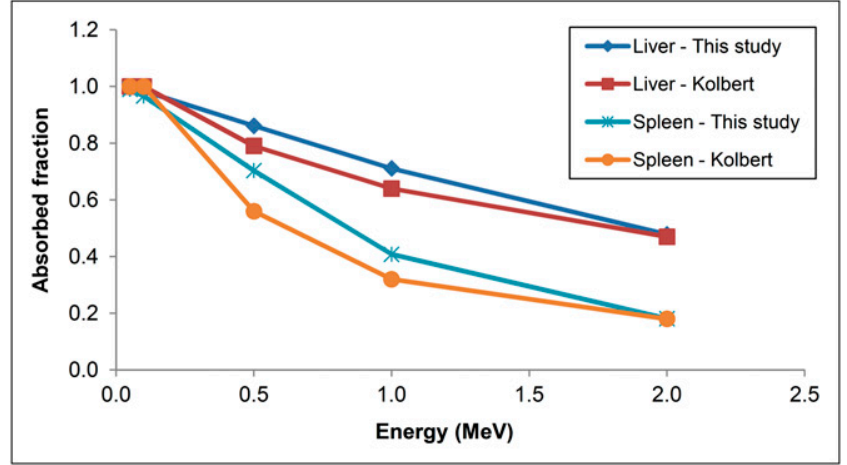

FIGURE 7. Comparison of self-absorbed fractions for monoenergetic electrons of various energies in this work (25-g mouse) and that of Kolbert et al. (8).

of the dose that the intestines will receive. Given the small dimensions of these models, it is doubtful that such extensive modeling of these small structures would produce reliable SAFs for separate compartments. During the estimation of DFs for hollow organs, standard assumptions about wall- or content-specific absorbed fractions may be applied, or a more uniform dose assumption may be used because of the small size of the organs. Which technique to use will be decided as we implement these models in software tools.

Figure 7 compares self-absorbed fractions for monoenergetic electron sources from this work with those reported by Kolbert et al. (8). The agreement is favorable, with values from this work being slightly higher, which is understandable because the organ masses in our models were larger than those of Kolbert et al. (8) (1.74 vs. $1.59 \mathrm{~g}$ for the liver and $0.111 \mathrm{vs} .0 .067 \mathrm{~g}$ for the spleen). Table 4 compares organ self-DFs for ${ }^{32} \mathrm{P}$ for liver and spleen between the 2 studies. Again, the comparison is favorable, with the differences being attributable to differences in organ masses. Table 5 compares organ masses (g) and self$\mathrm{DFs}(\mathrm{mGy} / \mathrm{MBq}-\mathrm{s})$ for several nuclides in the approximately 300 -g rat, between this work and that of Konijnenberg et al. (5) and Stabin et al. (10). Most differences can be explained by the differences in mass, except for the values of Konijnenberg et al. (5) for ${ }^{111} \mathrm{In}$.

\section{CONCLUSION}

Preclinical investigations with PET/CT or SPECT/CT image data can be used, as with clinical investigations, for calculating average doses to organs in small animals

TABLE 4. Comparison of Self-DFs (mGy/MBq-s) for ${ }^{32} \mathrm{P}$

$\begin{array}{lcc}\text { Organ } & \text { This study (25-g mouse) } & \text { Kolbert et al. (8) } \\ \text { Liver } & 5.2 \times 10^{-2} & 5.6 \times 10^{-2} \\ \text { Spleen } & 5.9 \times 10^{-1} & 7.6 \times 10^{-1}\end{array}$


TABLE 5. Comparison of Organ Masses (g) and Self-DFs (mGy/MBq-s) for Several Nuclides in Approximately 300-g Rat

\begin{tabular}{cccc}
$\begin{array}{c}\text { Organ } \\
\text { Mass (g) }\end{array}$ & This study & $\begin{array}{c}\text { Konijnenberg } \\
\text { et al. (5) }\end{array}$ & $\begin{array}{c}\text { Stabin } \\
\text { et al. (10) }\end{array}$ \\
Liver & 11.2 & 21.9 & 9.64 \\
Spleen & 0.884 & 0.80 & 0.42 \\
Lungs & 0.884 & 1.6 & 1.92 \\
Total body & 335 & 312 & 248 \\
90Y self-DFs & & & \\
Liver & $1.0 \times 10^{-2}$ & $5.9 \times 10^{-3}$ & $1.2 \times 10^{-2}$ \\
Spleen & $1.0 \times 10^{-1}$ & $1.1 \times 10^{-1}$ & $1.8 \times 10^{-1}$ \\
Lungs & $4.1 \times 10^{-2}$ & $1.8 \times 10^{-2}$ & $3.0 \times 10^{-2}$ \\
111 In self-DFs & & & \\
Liver & $5.9 \times 10^{-4}$ & $3.6 \times 10^{-4}$ & $7.9 \times 10^{-4}$ \\
Spleen & $6.1 \times 10^{-3}$ & $8.6 \times 10^{-3}$ & $1.3 \times 10^{-2}$ \\
Lungs & $4.8 \times 10^{-3}$ & $4.4 \times 10^{-3}$ & $2.7 \times 10^{-3}$ \\
177Lu self-DFs & & & \\
Liver & $2.1 \times 10^{-3}$ & $1.1 \times 10^{-3}$ & $\mathrm{NA}$ \\
Spleen & $2.6 \times 10^{-2}$ & $2.8 \times 10^{-2}$ & $\mathrm{NA}$ \\
Lungs & $2.3 \times 10^{-2}$ & $7.5 \times 10^{-3}$ & $\mathrm{NA}$ \\
& & & \\
\hline NA = not applicable. & & \\
\hline
\end{tabular}

represented by the models given here. The models may be used with simulated external radiation sources for dose calculations as well. Further models can be developed representing other animal species and used with the methods shown here.

\section{ACKNOWLEDGMENTS}

This work was supported partially by grant 1R42CA115122-01 from the National Institutes of Health.

\section{REFERENCES}

1. Hui TE, Fisher DR, Kuhn JA, et al. A mouse model for calculating cross-organ beta doses from yttrium-90-labeled immunoconjugates. Cancer. 1994;73(suppl): 951-957.

2 Yoriyaz H, Stabin M. Electron and photon transport in a model of a $30 \mathrm{~g}$ mouse [abstract]. J Nucl Med. 1997;38(suppl):228P.

3. Muthuswamy MS, Roberson PL, Buchsbaum DJ. A mouse bone marrow dosimetry model. J Nucl Med. 1998;39:1243-1247.
4. Flynn AA, Green AJ, Pedley RB, Boxer GM, Boden R, Begent RH. A mouse model for calculating the absorbed beta-particle dose from ${ }^{131} \mathrm{I}$ - and ${ }^{90} \mathrm{Y}$-labeled immunoconjugates, including a method for dealing with heterogeneity in kidney and tumor. Radiat Res. 2001;156:28-35.

5. Konijnenberg MW, Bijster M, Krenning EP, de Jong M. A stylized computational model of the rat for organ dosimetry in support of preclinical evaluations of peptide receptor radionuclide therapy with ${ }^{90} \mathrm{Y},{ }^{111} \mathrm{In}$, or ${ }^{177} \mathrm{Lu}$. J Nucl Med. 2004;45:1260-1269.

6. Petoussi-Henss N, Zanki M, Fill U, et al. The GSF family of voxel phantoms. Phys Med Biol. 2002;47:89-106.

7. Hindorf C, Ljungberg M, Strand S-E. Evaluation of parameters influencing S values in mouse dosimetry. J Nucl Med. 2004;45:1960-1965.

8. Kolbert KS, Watson T, Matei C, Xu S, Koutcher JA, Sgouros G. Murine S factors for liver, spleen and kidney. J Nucl Med. 2003;44:784-791.

9. Sgouros G, Chiu S, Pentlow KS, et al. Three-dimensional dosimetry for radioimmunotherapy treatment planning. J Nucl Med. 1993;34:1595-1601.

10. Stabin MG, Peterson TE, Holburn GE, Emmons MA. Voxel-based mouse and rat models for internal dose calculations. J Nucl Med. 2006;47:655-659.

11. Segars JP. Development and Application of the New Dynamic NURBS-Based Cardiac-Torso (NCAT) Phantom [Ph.D. dissertation]. Chapel Hill, NC: The University of North Carolina at Chapel Hill; 2001.

12. Segars WP, Lalush DS, Tsui BMW. Development of an interactive software application to model patient populations in the spline-based MCAT phantom. IEEE Nucl Sci Symp Conf Rec. 2000;3:20/51-20/55.

13. Segars WP, Tsui B. 4D MOBY and NCAT phantoms for medical imaging simulation of mice and men [abstract]. J Nucl Med. 2007;48(suppl 2):203P.

14. Zankl M, Petoussi-Henss N, Fill U, Regulla D. The application of voxel phantoms to the internal dosimetry of radionuclides. Radiat Prot Dosimetry. 2003; 105:539-548.

15. Agostinelli S, Allison $\mathrm{J}$, Amakoe $\mathrm{K}$, et al. $\mathrm{G}_{\text {eant }} 4$ : a simulation toolkit. $\mathrm{Nucl}$ Instru Meth A. 2003;506:250-303.

16. Cristy M, Eckerman K. Specific Absorbed Fractions of Energy at Various Ages from Internal Photons Sources. ORNL/TM-8381 V1-V7. Oak Ridge, TN: Oak Ridge National Laboratory; 1987.

17. International Commission on Radiological Protection (ICRP). Basic Anatomical and Physiological Data for Use in Radiological Protection: Reference Values. ICRP publication 89. New York, NY: Pergamon Press; 2003.

18. Stabin MG. Uncertainties in internal dose calculations for radiopharmaceuticals. J Nucl Med. 2008;49:853-860.

19. Stabin MG, da Luz CQPL. New decay data for internal and external dose assessment. Health Phys. 2002;83:471-475.

20. Stabin MG. Fundamentals of Nuclear Medicine Dosimetry. New York, NY: Springer; February 2008.

21. Bielajew A, Rogers D. PRESTA: the parameter reduced electron-step transport algorithm for electron Monte Carlo transport. Nucl Instrum Methods. 1987;B18: 165-181.

22. Briesmeister JF, ed. MCNP: A General Monte Carlo N-Particle Transport Code, Version 4C. LA-13709-M. Los Alamos, NM: Los Alamos National Laboratory; April 2000.

23. International Commissionon Radiological Protection. Human Alimentary Tract Model for Radiological Protection. ICRP publication 100. Oxford, England: Elsevier; 2006.

24. Snyder W, Ford M, Warner G. Estimates of Specific Absorbed Fractions for Photon Sources Uniformly Distributed in Various Organs of a Heterogeneous Phantom. MIRD pamphlet no. 5, rev. New York, NY: The Society of Nuclear Medicine; 1978. 